\title{
Mass Conservation and Inference of Metabolic Networks from High-Throughput Mass Spectrometry Data
}

\author{
PRADEEP BANDARU, ${ }^{1,2}$ MUKESH BANSAL, ${ }^{1}$ and ILYA NEMENMAN ${ }^{3}$
}

\begin{abstract}
We present a step towards the metabolome-wide computational inference of cellular metabolic reaction networks from metabolic profiling data, such as mass spectrometry. The reconstruction is based on identification of irreducible statistical interactions among the metabolite activities using the ARACNE reverse-engineering algorithm and on constraining possible metabolic transformations to satisfy the conservation of mass. The resulting algorithms are validated on synthetic data from an abridged computational model of Escherichia coli metabolism. Precision rates upwards of $50 \%$ are routinely observed for identification of full metabolic reactions, and recalls upwards of $20 \%$ are also seen.
\end{abstract}

Key words: algorithms, functional genomics, machine learning, reverse engineering, statistics.

\section{INTRODUCTION}

$\mathbf{P}$ Rior to the widesPREad aVAilability of anNotated metabolic Databases, metabolic network reconstruction was carried out primarily with biochemical assays of enzymatic activity (Schuster et al., 1999; Francke et al., 2005; Karp et al., 2002) resulting in a pathway-centric depiction of chemical reactions occurring in a cell. For some organisms, it has been possible to assemble these data into genome-wide metabolic networks by means of various Metabolic Flux Analysis (MFA) techniques (Schilling et al., 2000; Kauffman et al., 2003; Förster et al., 2003; Bonarius et al., 1997; Edwards and Palsson, 2000; Edwards et al., 2001). Now, recent developments in the burgeoning field of metabolic profiling (Steinhauser and Kopka, 2007; Trethewey et al., 1999; Saghatelian et al., 2004) and especially mass-spectrometry metabolomics (Enders et al., 2010; Rabinowitz, 2007; De Vos et al., 2007; Bennett et al., 2009; Ideker et al., 2001), which aims at high-throughput, real-time characterization of the entire cellular metabolic state, have opened up yet another way of approaching the metabolic network reconstruction problem, focusing on statistical interactions among metabolites. This parallels the transition that had happened in the analysis of transcriptional regulatory networks with the advent of gene expression profiling (Friedman, 2004; Margolin et al., 2006a,b; Faith et al., 2007; Bansai et al., 2007; Ideker et al., 2001), which similarly characterizes the genome-wide transcriptional state of the cell.

Specifically, distinct cellular phenotypes, phases of the cell cycle, or intrinsic and extrinsic perturbations result in changes in cellular metabolite concentrations. However, even with such changes, concentrations of

\footnotetext{
${ }^{1}$ Center for Computational Biology and Bioinformatics, Joint Centers for Systems Biology, and Columbia Initiative in Systems Biology, and ${ }^{2}$ Department of Biomedical Informatics, Columbia University, New York, New York.

${ }^{3}$ Departments of Physics and Biology and Computational and Life Sciences Strategic Initiative, Emory University, Atlanta, Georgia.
} 
metabolites that transform into each other should stay correlated, and the observed dependencies can be used to predict metabolic reactions computationally even if the identities of the metabolites are unknown, preventing the application of MFA methods. We attempted this approach (Nemenman et al., 2007) using the ARACNE statistical reverse engineering method, first developed in the context of inferring transcriptional networks from mRNA expression profiles (Margolin et al., 2006a,b; Basso et al., 2005). However, mass-spectrometry methods provide a wealth of information about the metabolites in addition to their abundances: MS-MS methods and isotopic labeling (Enders et al., 2010; Rabinowitz, 2007; De Vos et al., 2007; Bennett et al., 2009; Ishi, 2007) can recover the molecular structure, and, especially crucial for this article, masses of metabolites are typically measured to the accuracy of $10^{-4} \ldots 10^{-5}$. Since mass must be conserved in any metabolic transformation, this information presents an additional source of data for reverse engineering methods that has not been widely used. Namely, a statistical dependence among metabolites can indicate an actual metabolic transformation only if the putative chemical reaction constructed from these metabolites conserves mass. This rule can be applied even when identities of metabolites are unknown, and therefore, it is especially useful for high-throughput global metabolic profiling where spectral peaks cannot necessarily be identified.

In this article, we present a Mass-Constrained adaptation of the ARACNE algorithm, ARACNE-MC, which should be considered as a first foray into the field, laying the foundation for future studies. Note that, in the case of metabolism, we are not content with knowing just the statistical dependencies among the metabolites, even if they correspond to bona-fide metabolic transformations. Instead we aim at a substantially more complicated task of reconstructing complete metabolic reactions, identifying all of their substrates and products.

We test the algorithm on reduced toy models of the Escherichia coli metabolome with in silico simulated metabolic profiles. Applications to real-world biological problems will have to wait for larger experimental datasets.

\section{RESULTS}

\subsection{Outlines of the algorithms}

2.1.1. The ARACNE algorithm. The basis of the reverse engineering approach we undertake is the notion that molecular species that participate in biochemical reactions have statistically dependent expressions (Nemenman et al., 2007). Within the ARACNE framework (Margolin et al., 2006a,b; Basso et al., 2005), one views metabolite expressions as random variables sampled from stationary probability distributions. This randomness accounts for effects of unknown states of unobserved metabolites and other chemical species and of the experimental noise. Chemical transformations correspond to nonzero multivariate statistical dependencies among metabolite concentrations (Margolin et al., 2010). In particular, the relevant measure of statistical dependency between two variables $\left(c_{1}, c_{2}\right)$ is their mutual information (MI), which is defined as in Cover and Thomas (1991)

$$
I\left[c_{1} ; c_{2}\right]=\left\langle\log _{2} \frac{P\left(c_{1}, c_{2}\right)}{P\left(c_{1}\right) P\left(c_{2}\right)}\right\rangle_{P\left(c_{1}, c_{2}\right)},
$$

where $P\left(c_{1}, c_{2}\right)$ denotes their joint probability distribution, $P\left(c_{i}\right)$ are the marginal probability distributions, and $\langle\ldots\rangle_{P}$ is the average over the distribution $P$.

The MI is generally nonzero for bona fide interacting metabolites, but it may also be nonzero for chemicals that are connected through an intermediate and do not transform into each other directly. In fact, such false positives are generally a bigger problem than false negatives (i.e., missing a true interaction) in computational networks reverse engineering: false positives are plentiful and lower the confidence in the validity of every specific prediction.

The ARACNE algorithm (Margolin et al., 2006a,b; Basso et al., 2005) eliminates some of the false positives by using the data processing inequality (DPI) (Cover and Thomas, 1991) to isolate statistical interactions that have the highest chance of corresponding to true biological transformations. Specifically, under certain assumptions that are often applicable in transcriptional (Margolin et al., 2005) and metabolic (Nemenman et al., 2007) contexts, if

$$
I\left[c_{1} ; c_{3}\right] \leq \min \left(I\left[c_{1} ; c_{2}\right], I\left[c_{2} ; c_{3}\right]\right),
$$


then the $c_{1} \leftrightarrow c_{3}$ interaction is indirect. Hence, ARACNE starts with a fully connected graph of the measured chemical species as putative interaction partners, compares MIs for every triplet of chemical species in the dataset, and removes the weakest pairwise interaction in every such triplet from further consideration (for details of the protocol, cf. Margolin et al., 2005). Practical complications in the application of ARACNE revolve around accurate, unbiased estimation of MI and of the threshold (5-15\% for typical applications) above which a difference between two MI values becomes significant for the DPI application (Margolin et al., 2006a,b). Though developed for reverse engineering transcriptional networks, ARACNE has also been validated in the context of synthetic metabolic networks (Nemenman et al., 2007).

2.1.2. The ARACNE-MC algorithm. As we have emphasized, mass-spectrometry provides additional information about the metabolic state of a cell, namely, masses of the metabolites. This creates extra means for eliminating false positive metabolic transformations beyond those tried in Nemenman et al. (2007): even if statistical dependencies suggest an interaction, the implied putative chemical reaction may not conserve mass and hence be impossible. To use this additional constraint, we propose the ARACNEMC algorithm (MC stands for Mass Constrained). Like the original ARACNE, ARACNE-MC aims at reducing false positives, potentially at the cost of increasing the false negatives.

For a list of metabolites $\mu_{a}$ with masses $m_{a}$ and metabolic activities in $i^{\prime}$ th spectrometer run $c_{a i}$, we start by building a list of putative metabolic reactions allowed by mass conservation, focusing on a limited set of template reactions that are allowed in the analysis. In this article, we consider only three templates (a) $1 \times 1$, $\mu_{l_{1}} \leftrightarrow \mu_{r_{2}}$; (b) $1 \times 2, \mu_{l_{1}} \leftrightarrow \mu_{r_{1}}+\mu_{r_{2}}$; and (c) $2 \times 2, \mu_{l_{1}}+\mu_{l_{2}} \leftrightarrow \mu_{r_{1}}+\mu_{r_{2}}$ (indexes $l$ and $r$ stand for left and right, respectively). See Figure 1 for example reactions of each template. Eliminating more complicated reactions from consideration will result in the elimination of bona fide statistical interactions, and hence in extra false negatives, which we accept. We build a list of all putative reactions that fall into the allowed template classes and satisfy the mass conservation, $\left|\sum m_{l_{i}}-\sum m_{r_{i}}\right| \leq \varepsilon \sum m_{l_{i}}$, where $\varepsilon$ is the mass equality tolerance, set to $\varepsilon=10^{-4}$ throughout this article. We refer to such reactions as conforming reactions. Identifying them has a computational complexity of $O\left(M^{\alpha}\right)$, where $\alpha$ is the maximum number of metabolites on either side of the template ( 2 in this article).

While possible in principle, a conforming reaction may not exist in a real cell due to a multitude of factors. If present, it should result in statistical dependencies among its reactants and products. There will be up to one such dependence for a $1 \times 1$ reaction (two choose two), three for a $1 \times 2$ reaction (three choose two), and six for a $2 \times 2$ reaction (four choose two). Therefore, we prune the list of conforming reactions by identifying those that are supported by statistical dependencies. To do so, we apply ARACNE to metabolite activity profiles, $c_{a i}$. Specifically, we estimate the pairwise MIs $I\left[c_{\mathrm{a}}, c_{b}\right]$ using the algorithms of Margolin et al. (2006a,b) and then apply the DPI to the list of MIs to select the interactions that have the highest chance of being direct. Then the conforming reactions are ranked by how many of their pairwise member metabolite interactions are identified as direct by ARACNE. We expect that reactions that are conforming
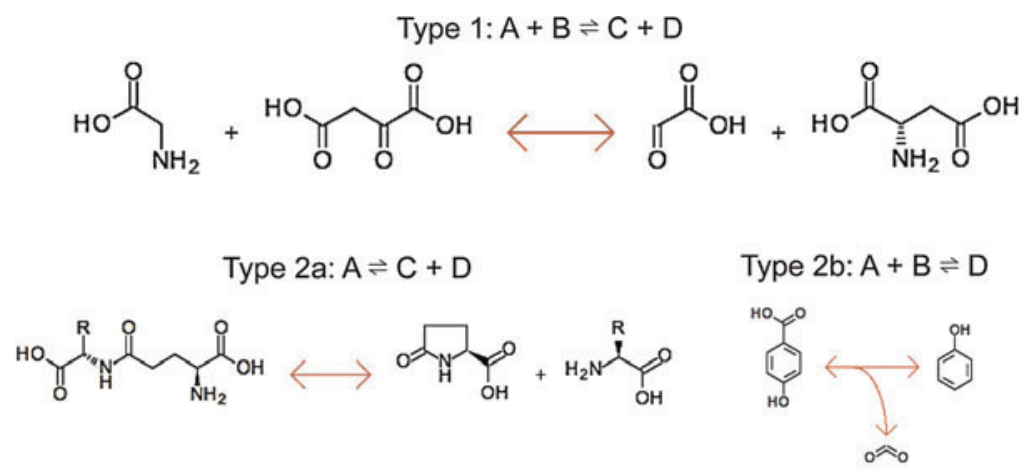

FIG. 1. Examples of three types of metabolic reactions included in the synthetic network and generated by the mass constraints algorithm. Type 1 reactions are termed twoby-two reactions, Type $2 \mathrm{a}$ and $2 \mathrm{~b}$ reactions are termed one-by-two reactions, and Type 3 reactions are termed one-by-one reactions. CheType 3: A $\rightleftharpoons D$ mical structures from KEGG are provided for illustration.

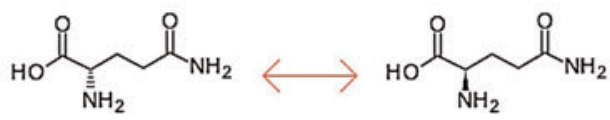


and supported statistically will have a high chance to be bona fide metabolic reactions. The ARACNE-MC1 algorithm thus requires selection of the threshold for the number of ARACNE-supported metabolite pairwise interactions, and identifies all of the conforming reactions passing the threshold as putative reactions.

We notice that the same metabolic statistical interaction may be a part of multiple reactions. One "strong" reaction may be responsible for much of the MI associated with a particular link, and thus for the corresponding interaction surviving the ARACNE DPI application. However, ARACNE-MC1 would count this interaction in support of every conforming reaction to which it belongs. To avoid this multiple counting, we sort all conforming reactions by their "strength" as measured by the cumulative mutual information in all of its interactions. We then ensure that an interaction is counted as supporting only the strongest of its associated reactions; this is the ARACNE-MC2 algorithm.

Flowcharts of both algorithms are illustrated in Figure 2. The algorithms, implemented in MATLAB, are available at http://menem.com/ ilya/wiki/index.php/Bandaru_et_al.,_2011. The performance of the algorithms will depend on a variety of choices, such as the DPI and mass comparison tolerances, MI estimation parameters, or thresholds for the number of interaction supports needed to proclaim a reaction as existing. Some of these choices are explored later in the article. For others, which we believe may differ dramatically for synthetic and for experimental data, we leave the detailed analysis to future publications.

\subsection{Synthetic tests of ARACNE-MC}

2.2.1. Data generation and performance metrics. To validate performance of ARACNE-MC, we used the Kyoto Encyclopedia of Genes and Genomes (KEGG) (Ogata et al., 1999) to create synthetic metabolic networks, which then served as a source of simulated data for tests. KEGG provides a detailed description of metabolites and reactions found in the metabolic pathways of various model organisms. The entirety of the metabolic pathways of Escherichia coli were downloaded and pruned to include only mass-balanced reactions of the three types shown in Figure 1. Two different synthetic networks were

FIG. 2. Flowchart of the ARACNE-MC algorithm.

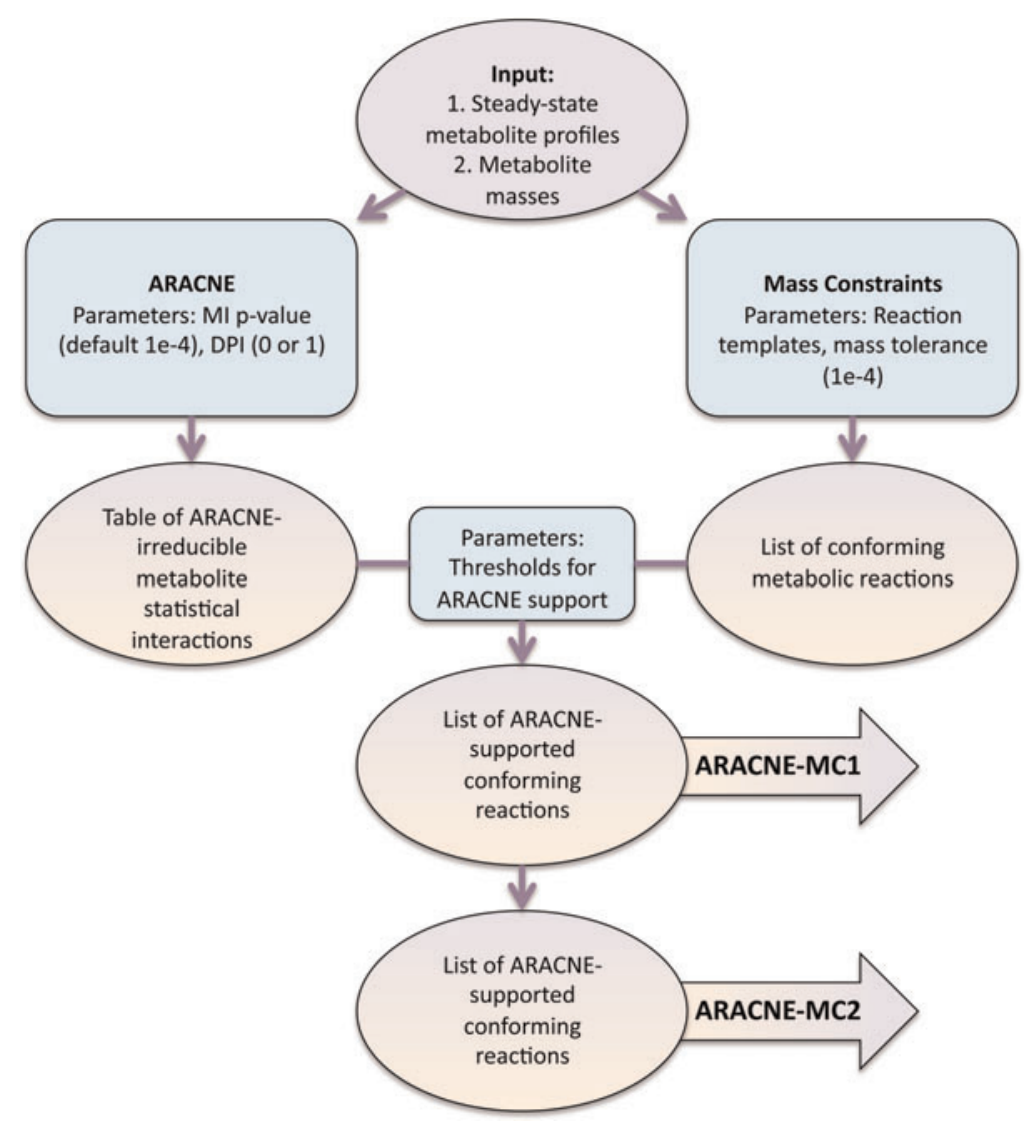


constructed to test the performance of ARACNE-MC on metabolic networks of different sizes: a small network, containing 86 unique metabolites and 50 metabolic reactions, and a large network with 218 metabolites and 136 reactions. Detailed specifications of the networks are available at http://menem.com/ 〜ilya/wiki/index.php/Bandaru_et_al.,_2011.

Since a majority of kinetic rates are unknown in KEGG, for each of the reactions we randomly generated forward and backward rates, with the forward to backward ratio being, on average, a hundred. We used the open-source COPASI software (Hoops et al., 2006) to simulate the dynamics of the network multiple times, perturbing the kinetic rates each time by a random multiplicative factor with a standard deviation of $15 \%$. This is similar to the approach in Margolin et al. $(2006 \mathrm{a}, \mathrm{b})$ and is supposed to represent changes to the rates due to different extracellular environments and phenotypic and metabolic states of the cells. Each simulation started with equal metabolite concentrations of $1 \mu \mathrm{M}$ and was run to a steady state. One thousand steady states were simulated this way and used as synthetic metabolic profiles for input to ARACNE-MC.

To measure the algorithm performance, we choose the metrics of precision and recall. Precision, $\pi=N_{T P} /\left(N_{T P}+N_{F P}\right)$, measures the fraction of true predictions among all predictions (where indices $T, F$, $P$, and $N$ stand for true, false, positives, and negatives). Precision corresponds to the expected success rate in the experimental validation of computational predictions. Similarly, recall, $\rho=N_{T P} /\left(N_{T P}+N_{F N}\right)$, indicates the fraction of all reactions recovered by the algorithm. Precision and recall values of 1 indicate perfect performance. However, there is generally a tradeoff between the two. We emphasize that all of these metrics are calculated based on recovery of complete metabolic reactions, rather than simple statistical correlations among the metabolites, as in Nemenman et al. (2007).

2.2.2. ARACNE-MC performance. We performed two tests to verify that accurate knowledge of both the metabolite profiles and the mass constraints is necessary for ARACNE-MC1 and 2 performance. Firstly, we randomized entries in the mass-conforming reactions while leaving the statistical relations intact. Secondly, we randomized metabolic profiles while preserving the correct mass constraints. In both cases, ARACNE-MC1 and 2 failed to predict metabolic reactions beyond chance, indicating an equal reliance on the two types of data (results not shown).

Table 1 details the results from the ARACNE-MC1 algorithm in the reconstruction of the large (218 metabolites) and small (86 metabolites) synthetic networks. Precisions well over 50\% with significant recall rates are seen for many parameter combinations. The results suggest that the ARACNE algorithm is robust to changes in network size and topology. We emphasize again that, for our performance metrics, all substrates and products of a reaction must be predicted correctly for the reaction to be counted as correct. This is a very stringent performance test.

Performance of ARACNE-MC2 is illustrated in Table 2. We see that removing multiple counting of interactions has an effect of increasing the precision (sometimes to the maximum level of 1) with only a marginal loss in the recall.

To illustrate the dependence of the ARACNE-MC reconstruction on the DPI tolerance parameter, Table 1 shows performance of ARACNE-MC1 for the tolerance of 1 (i.e., no DPI applied). While performance is weaker compared to the tolerance of 0 , the effect is not dramatic, suggesting weak sensitivity to

Table 1. Performance of ARACNE-MC1 for Different Networks and DPI Tolerances

\begin{tabular}{lccccc}
\hline Template & ARACNE interactions & $T P$ & $F P$ & Precision & Recall \\
\hline $1 \times 1$ & 1 & $1 / 0 / 15$ & $4 / 0 / 11$ & $0.20 / 0 / 0.58$ & $0.50 / 0 / 0.71$ \\
$2 \times 1$ & $2 / 7 / 6$ & $1 / 8 / 10$ & $0.67 / 0.47 / 0.38$ & $0.11 / 0.37 / 0.16$ \\
& 3 & $6 / 14 / 20$ & $2 / 17 / 33$ & $0.75 / 0.45 / 0.38$ & $0.32 / 0.74 / 0.53$ \\
& 2 & $0 / 5 / 9$ & $0 / 21 / 2$ & $0 / 0.19 / 0.82$ & $0 / 0.17 / 0.12$ \\
& 6 & $1 / 10 / 14$ & $3 / 31 / 2$ & $0.25 / 0.24 / 0.88$ & $0.03 / 0.34 / 0.18$ \\
& 5 & $4 / 18 / 20$ & $6 / 98 / 29$ & $0.40 / 0.16 / 0.41$ & $0.14 / 0.62 / 0.26$ \\
& 4 & $12 / 23 / 48$ & $28 / 369 / 355$ & $0.30 / 0.06 / 0.12$ & $0.41 / 0.79 / 0.62$ \\
& 3 & $21 / 28 / 61$ & $227 / 969 / 77$ & $0.08 / 0.03 / 0.02$ & $0.72 / 0.97 / 0.79$ \\
\hline
\end{tabular}

The first value in each cell in the four right columns corresponds to the small network with zero tolerance; the second value is for the small network with the tolerance of one; and the third value is for the large network. All data is for 1000 simulated metabolic profiles. When the DPI tolerance is 0 , precisions over $80 \%$ are possible with recalls in the teens when large ARACNE support for a conforming reaction is requested, and recall of $25-50 \%$ still leaves the precision around $40 \%$. 
Table 2. Performance of ARACNE-MC2 for the Small and the Large Networks

\begin{tabular}{|c|c|c|c|c|c|c|}
\hline Template & $\begin{array}{c}\text { ARACNE } \\
\text { interactions }\end{array}$ & $\begin{array}{l}\text { Surviving ARACNE } \\
\quad \text { interactions }\end{array}$ & $T P$ & $F P$ & Precision & Recall \\
\hline $1 \times 1$ & 1 & N/A & $0 / 0$ & $0 / 0$ & $0 / 0$ & $0 / 0$ \\
\hline \multirow[t]{5}{*}{$2 \times 1$} & 3 & 3 & $1 / 3$ & $0 / 0$ & $1 / 1$ & $0.5 / 0.8$ \\
\hline & & 2 & $2 / 6$ & $0 / 0$ & $1 / 1$ & $0.11 / 0.16$ \\
\hline & & 1 & $2 / 6$ & $0 / 0$ & $1 / 1$ & $0.11 / 0.16$ \\
\hline & 2 & 2 & $1 / 5$ & $0 / 0$ & $1 / 1$ & $0.05 / 0.13$ \\
\hline & & 1 & $3 / 12$ & $0 / 0$ & $1 / 1$ & $0.16 / 0.32$ \\
\hline \multirow[t]{13}{*}{$2 \times 2$} & 6 & 6 & $0 / 5$ & $0 / 2$ & $0 / 0.71$ & 0/0.06 \\
\hline & & 5 & $0 / 9$ & $0 / 2$ & $0 / 0.82$ & $0 / 0.12$ \\
\hline & 5 & 5 & $0 / 14$ & $0 / 2$ & $0 / 0.88$ & $0 / 0.28$ \\
\hline & & 4 & $0 / 14$ & $0 / 2$ & $0 / 0.88$ & $0 / 0.28$ \\
\hline & & 3 & $0 / 14$ & $0 / 2$ & $0 / 0.88$ & $0 / 0.28$ \\
\hline & 4 & 4 & $3 / 12$ & $7 / 14$ & $0.43 / 0.46$ & $0.1 / 0.16$ \\
\hline & & 3 & $4 / 19$ & $9 / 16$ & $0.44 / 0.54$ & $0.14 / 0.25$ \\
\hline & & 2 & $4 / 20$ & $10 / 18$ & $0.4 / 0.53$ & $0.14 / 0.26$ \\
\hline & 3 & 3 & $8 / 21$ & $17 / 40$ & $0.47 / 0.34$ & $0.28 / 0.27$ \\
\hline & & 2 & $10 / 35$ & $28 / 62$ & $0.36 / 0.36$ & $0.35 / 0.45$ \\
\hline & & 1 & $10 / 38$ & $34 / 91$ & $0.29 / 0.29$ & $0.35 / 0.49$ \\
\hline & 2 & 2 & $10 / 22$ & $66 / 178$ & $0.15 / 0.11$ & $0.34 / 0.29$ \\
\hline & & 1 & $13 / 41$ & $113 / 305$ & $0.12 / 0.12$ & $0.45 / 0.53$ \\
\hline
\end{tabular}

The first value in each cell in the four right columns corresponds to the small network and the second value corresponds to the large network. The DPI tolerance for both networks is 0 . Note an increase in precision and/or recall for various template and interaction thresholds.

the parameters. The specific best value of the parameter will likely depend on the size of the dataset and on the experimental noise, and will need to be established for each particular application independently as in Wang et al. (2009).

Finally, a crucial feature of any computational reverse engineering algorithm is the dependence of its performance on the size of the experimental dataset. We test this for ARACNE-MC2 in Table 3. Specifically, both the precision and the recall degrade gracefully as the data set size decreases from 1000 to 100 , and no meaningful reconstruction is possible when the size becomes comparable to the number of the analyzed metabolic species. This explains, in particular, why our application of the algorithms to the existing experimental data set of Ishii et al. (2007), which includes approximately 30 steady-state metabolic profiles of Escherichia coli and 195 metabolites in each profile, has failed.

\section{DISCUSSION}

The ARACNE-MC1 and 2 algorithms represent the first computational step towards identification of metabolic reaction networks from high-throughput mass-spectrometry profile data, armed with detailed

Table 3. Performance for Selected Template and Interaction Thresholds from the Large Network Against a Varying Number of Input Metabolic Profiles Using the ARACNE-MC2 Algorithm

\begin{tabular}{|c|c|c|c|c|}
\hline \multirow{2}{*}{$\begin{array}{c}\text { Number of } \\
\text { samples }\end{array}$} & \multicolumn{2}{|c|}{$2 \times 1 / 3 / 2$} & \multicolumn{2}{|c|}{$2 \times 2 / 4 / 3$} \\
\hline & Precision & Recall & Precision & Recall \\
\hline 1000 & 1 & 0.16 & 0.54 & 0.25 \\
\hline 500 & 1 & 0.13 & 0.58 & 0.25 \\
\hline 250 & 1 & 0.13 & 0.43 & 0.19 \\
\hline 100 & 1 & 0.03 & 0 & 0 \\
\hline
\end{tabular}

Generally, precision and recall decrease with fewer input metabolite profiles. 
knowledge of metabolite masses. Performance of the algorithm on synthetic data sets is encouraging, warranting further development and application to real-life data sets, when available. Selection of optimal values of many parameters of the algorithm, which we expect to depend on the details of the experimental data, will need to be performed at that time. Further, depending on the experimental resolution for many small, common metabolites, additional modifications of ARACNE-MC will need to be considered. In particular, to reduce the rate of false negatives, frequent interactions among common substrates (ATP, water, NADP, etc.) can be treated as supported statistically for every conforming reaction.

As implemented now, the algorithm is data-intensive, requiring more metabolic profiles than the number of considered metabolites. Current absence of such large datasets is the biggest obstacle in application of the algorithm to real-world problems. However, we expect that ion-mobility mass spectrometry with nanoliter chemostat cultures (Enders et al., 2010) will be able to provide the necessary amounts of data in the immediate future.

\section{METHODS}

\subsection{Synthetic networks generation}

Synthetic metabolic networks were created from the KEGG database. Using Escherichia coli as a model system for these synthetic networks, we downloaded mass-balanced reactions randomly, selecting reactions from the entirety of Escherichia coli metabolic pathways, so that the final analyzed network is representative of the metabolism of E. coli. A small synthetic network containing 86 unique metabolites and 50 metabolic reactions and a large synthetic network containing 218 metabolites and 136 metabolic reactions were generated. See the author's website for detailed descriptions of the metabolites in each network and their corresponding masses.

\subsection{Analysis and simulation parameters}

Two main parameters of ARACNE algorithm are the p-value for accepting an MI estimate as nonzero and the DPI tolerance threshold. For the purposes of this study, the DPI tolerance was varied between 1 (no DPI application) and 0 (stringent edge elimination), and the p-value threshold was set to the default level of 1e-4. Additionally, the mass comparison relative tolerance was 1e-4.

\section{ACKNOWLEDGMENTS}

We especially thank Andrea Califano, who has been an invaluable help in the early stages of this research. We also thank William Hlavacek, Fangping Mu, Joel Berendzen, and Manjunath Kustagi for important contributions. P.B. and I.N. were partially supported by NIH/NIGMS and NIH/NCI (grants 1R21GM080216 and 1R01CA132629).

\section{DISCLOSURE STATEMENT}

No competing financial interests exist.

\section{REFERENCES}

Bansal, M., Belcastro, V., Ambesi-Impiombato, A., et al. 2007. How to infer gene networks from expression profiles. Mol. Syst. Biol. 3, 78.

Basso, K., Margolin, A.A., Stolovitzky, G., et al. 2005. Reverse engineering of regulatory networks in human B cells. Nat. Genet. 37, 382-390.

Bennett, B.D., Kimball, E.H., Gao, M., et al. 2009. Absolute metabolite concentrations and implied enzyme active site occupancy in Escherichia coli. Nat. Chem. Biol. 5, 593-599.

Bonarius, H., Schmid, G., and Tramper, J. 1997. Flux analysis of underdetermined metabolic networks: the quest for the missing constraints. Trends Biotechnol. 15, 308-314. 
Cover, T.M., and Thomas, J.A. 1991. Elements of Information Theory. John Wiley \& Sons, New York.

De Vos, R.C.H., Moco, S., Lommen, A., et al. 2007. Untargeted large-scale plant metabolomics using liquid chromatography coupled to mass spectrometry. Nat. Protoc. 2, 778-791.

Edwards, J.S., and Palsson, B.O. 2000. The Escherichia coli MG1655 in silico metabolic genotype: its definition, characteristics, and capabilities. Proc. Natl. Acad. Sci. USA 97, 5528-5533.

Edwards, J.S., Ibarra, R.U., and Palsson, B.O. 2001. In silico predictions of Escherichia coli metabolic capabilities are consistent with experimental data. Nat. Biotechnol. 19, 125-130.

Enders, J.R., Marasco, C.C., Kole, A., et al. 2010. Towards monitoring real-time cellular response using an integrated microfluidics-matrix assisted laser desorption ionisation/nanoelectrospray ionisation-ion mobility-mass spectrometry platform. IET Syst. Biol. 4, 416.

Faith, J.J., Hayete, B., Thaden, J.T., et al. 2007. Large-scale mapping and validation of Escherichia coli transcriptional regulation from a compendium of expression profiles. PLoS Biol. 5, e8.

Förster, J., Famili, I., Fu, P., et al. 2003. Genome-scale reconstruction of the Saccharomyces cerevisiae metabolic network. Genome Res. 13, 244-253.

Francke, C., Siezen, R.J., and Teusink, B. 2005. Reconstructing the metabolic network of a bacterium from its genome. Trends Microbiol. 13, 550-558.

Friedman, N. 2004. Inferring cellular networks using probabilistic graphical models. Science 303, 799-805.

Hoops, S., Sahle, S., Gauges, R., et al. 2006. COPASI-a COmplex PAthway SImulator. Bioinformatics 22, 30673074.

Ideker, T., Galitski, T., and Hood, L. 2001. A new approach to decoding life: systems biology. Annu. Rev. Genomics Hum. Genet. 2, 343-372.

Ishii, N., Nakahigashi, K., Baba, T., et al. 2007. Multiple high-throughput analyses monitor the response of E. coli to perturbations. Science 316, 593-597.

Karp, P.D., Paley, S., and Romero, P. 2002. The Pathway Tools software. Bioinformatics 18, Suppl 1, S225-S232.

Kauffman, K.J., Prakash, P., and Edwards, J.S. 2003. Advances in flux balance analysis. Curr. Opin. Biotechnol. 14, 491-496.

Margolin, A.A., Nemenman, I., Basso, K., et al. 2006a. ARACNE: an algorithm for the reconstruction of gene regulatory networks in a mammalian cellular context. BMC Bioinformatics 7, Suppl 1, S7.

Margolin, A.A., Wang, K., Lim, W.K., et al. 2006b. Reverse engineering cellular networks. Nat. Protoc. 1, $662-671$.

Margolin, A.A., Wang, K., Califano, A., et al. 2010. Multivariate dependence and genetic networks inference. IET Syst. Biol. 4, 428.

Nemenman, I., Escola, G.S., Hlavacek, W.S., et al. 2007. Reconstruction of metabolic networks from high-throughput metabolite profiling data: in silico analysis of red blood cell metabolism. Ann. N. Y. Acad. Sci. 1115, 102-115.

Ogata, H., Goto, S., Sato, K., et al. 1999. KEGG: Kyoto Encyclopedia of Genes and Genomes. Nucleic Acids Res. 27, 29-34.

Rabinowitz, J.D. 2007. Cellular metabolomics of Escherchia coli. Expert Rev. Proteomics 4, 187-198.

Saghatelian, A., Trauger, S.A., Want, E.J., et al. 2004. Assignment of endogenous substrates to enzymes by global metabolite profiling. Biochemistry 43, 14332-14339.

Schilling, C.H., Edwards, J.S., Letscher, D., et al. 2000. Combining pathway analysis with flux balance analysis for the comprehensive study of metabolic systems. Biotechnol. Bioeng. 71, 286-306.

Schuster, S., Dandekar, T., and Fell, D.A. 1999. Detection of elementary flux modes in biochemical networks: a promising tool for pathway analysis and metabolic engineering. Trends Biotechnol. 17, 53-60.

Steinhauser, D., and Kopka, J. 2007. Methods, applications and concepts of metabolite profiling: primary metabolism. EXS 97, 171-194.

Trethewey, R.N., Krotzky, A.J., and Willmitzer, L. 1999. Metabolic profiling: a Rosetta Stone for genomics? Curr. Opin. Plant Biol. 2, 83-85.

Wang, K., Saito, M., Bisikirska, B.C., et al. 2009. Genome-wide identification of post-translational modulators of transcription factor activity in human B cells. Nat. Biotechnol. 27, 829-837.

Address correspondence to: Dr. Ilya Nemenman

Departments of Physics and Biology Computational and Life Sciences Strategic Initiative

Emory University

Atlanta, GA 30322

E-mail: ilya.nemenman@emory.edu 